\title{
Anatomía floral comparativa del género Polianthes (Agavaceae)
}

\section{Comparative floral anatomy of the genus Polianthes (Agavaceae)}

\author{
Héctor Serrano-Casas ${ }^{1}$, Eloy Solano $^{1 *}$ y Teresa Terrazas ${ }^{2}$ \\ ${ }^{l}$ Facultad de Estudios Superiores Zaragoza, Unidad de Investigación en Sistemática Vegetal y Suelo, Herbario FEZA, Universidad Nacional Autónoma \\ de México, Apartado postal 9-020, 09230, México, D. F., México. \\ ${ }^{2}$ Departamento de Botánica, Instituto de Biología, Universidad Nacional Autónoma de México, Apartado postal 70-633, 04510, México, D. F., México. \\ *Correspondencia: solanoec@correo.unam.mx
}

\begin{abstract}
Resumen. Se realizó un estudio anatómico de las flores de algunas especies en los subgéneros Polianthes y Bravoa del género Polianthes L. (Agavaceae), con el propósito de investigar si la actual clasificación subgenérica es adecuada. Los taxa analizados del subgénero Polianthes fueron P. densiflora, P. nelsonii y P. platyphylla, y del subgénero Bravoa, P. geminiflora var. geminiflora, P. howardii y P. multicolor. Las características anatómicas florales entre las especies son similares y comparten con otros miembros de la familia Agavaceae los nectarios septales y los óvulos anátropos, bitégmicos y crasinucelados. En los taxa del subgénero Bravoa, los filamentos se originan en la base del tubo floral, mientras que en el subgénero Polianthes se originan por debajo de los tépalos internos del perianto. Anteriormente, sin considerar la anatomía de las flores, se planteaba que los filamentos se originaban en ambos subgéneros desde la base del tubo del perianto y que éstos permanecían adnados al mismo, separándose a diferentes niveles. En el nivel interespecífico, P. howardii presenta nectarios de mayor longitud que los lóculos, en relación con los de las otras especies estudiadas.
\end{abstract}

Palabras clave: Bravoa, filamentos, nectarios septales, óvulos anátropos, polen abortivo, rafidios.

\begin{abstract}
An anatomical floral study of some species of the genus Polianthes (Agavaceae), of both subgenus Polianthes and subgenus Bravoa (Agavaceae) was carried out, with the aim of testing the adequacy of the present subgeneric classification. The taxa studied of subgenus Polianthes were P. densiflora, P. nelsonii and P. platyphylla; and P. geminiflora var. geminiflora, P. howardii and P. multicolor of subgenus Bravoa. The anatomical characters between these species are similar, sharing with other members of the Agavaceae family the septal nectaries and the anatropous, bitegmic, and crassinucellated ovules. In the taxa of subgenus Bravoa, the filaments originate from the floral tube base, while in subgenus Polianthes they originate near the base of the internal tepals from the perianth tube. Before this anatomical study, it was considered that stamens originated in both subgenera at the base of the perianth tube, with their filaments remaining adnate to the perianth tube, getting separated at different levels. At interspecific level, the nectary/locule length ratio in $P$. howardii is larger than in the rest of the studied species.
\end{abstract}

Key words: Bravoa, filaments, septal nectaries, anatropous ovules, abortive pollen, raphides.

\section{Introducción}

El género Polianthes pertenece a la familia Agavaceae (Dahlgren et al., 1985), es endémico de México y su límite de distribución norte se encuentra en Chihuahua y Tamaulipas; hacia el sur llega hasta el norte de Oaxaca (Solano y Feria, 2007). El número de especies de este género ha variado en función de los criterios utilizados para su delimitación; de este modo, Rose (1903) reconoció 12 especies, Espejo y López (1992) 15, García-

Recibido: 26 junio 2009; aceptado: 25 junio 2010
Mendoza y Galván (1995) 13 y Solano y Feria (2007) 14 especies y 16 taxa. En un análisis cladístico de los géneros Polianthes, Bravoa y Pseudobravoa, con base en caracteres morfológicos y anatómicos vegetativos, Solano (2000) reconoció 14 especies, incluidas en 2 subgéneros, Bravoa y Polianthes. Este autor menciona como caracteres distintivos de Bravoa que la inflorescencia es un racimo con 2 flores por nudo (excepto en $P$. howardii), colgantes a cercanamente horizontales, color coral, anaranjado, anaranjado-verdoso, anaranjado-amarillento, rojo, rojoverdoso, rosado con rayas blancas o rara vez blanco, los tépalos cortos, erectos, ovados a deltoides, orbiculares a transversalmente elípticos; estambres insertos desde 2 a 5 
$\mathrm{mm}$ y hasta $19 \mathrm{~mm}$ por arriba del ápice del ovario. Para Polianthes reconoce que la inflorescencia es una espiga con 2 flores por nudo (excepto en $P$. densiflora, con 1 flor por nudo), la porción distal del tubo es horizontal, cercanamente vertical o ligeramente curvada, de color amarillo, blanco a rosado y hasta rojo con la edad; los tépalos cortos o largos, erectos o reflexos, ovados, oblongos o elípticos. Estambres insertos en el tubo, 2 a $3 \mathrm{~mm}$ por debajo de la base de los tépalos.

Los únicos estudios sobre anatomía de las flores de Polianthes fueron realizados por Joshi y Pantulu (1941), Pantil y Pai (1985) y Álvarez $(1987,1988)$ en P. tuberosa. Joshi y Pantulu (1941) estudiaron la organogénesis de la flor con la finalidad de determinar el origen del ovario ínfero; además, analizaron la vascularización de los verticilos florales. Pantil y Pai (1985) registraron que la abertura de los nectarios se localiza en la porción distal del ovario y Álvarez $(1987,1988)$ la ubicó sobre el estilo, cercana al estigma. Con el objetivo de identificar caracteres anatómicos que apoyen la propuesta de reconocer a los subgéneros Bravoa y Polianthes, en el presente estudio se revisó la anatomía floral de 6 especies del género que representan la mayor variabilidad floral del mismo.

\section{Materiales y métodos}

Se seleccionó material de los siguientes especímenes recolectados por Solano et al., y preservados en GAA (glicerina-alcohol etílico-agua 1:2:3): Polianthes densiflora (B. L. Rob. et Fernald) Shinners, E. Solano C. et al. 892; P. geminiflora (Lex.) Rose var. geminiflora, E. Solano C. 790; P. howardii Verh-Will., E. Solano C. y C. Correa D. 868; P. multicolor E. Solano et Dávila, E. Solano C. y C. Correa D. 870, 1112; P. nelsonii Rose, E. Solano C. y C. Correa D. 1871, 1882; P. platyphylla Rose, E. Solano C. 798, 830, R. Ríos G. y E. Solano C.149. Las recolectas se realizaron de 1994 a 2006 en varias localidades de la distribución del género, durante el periodo de floración (junio a octubre). Los ejemplares herborizados fueron determinados taxonómicamente con la ayuda de bibliografía especializada y depositados en el herbario FEZA, con un duplicado en el herbario Nacional (MEXU).

Los botones florales se agruparon de acuerdo con su tamaño. Estos botones se incluyeron en parafina Paramex $\mathrm{y}$ fueron seccionados transversal y longitudinalmente (10-12 $\mu \mathrm{m}$ de grosor) con un micrótomo rotatorio, marca Leica, modelo RM2125 RT. Las secciones se tiñeron con safranina-verde rápido y se montaron con resina sintética (Johansen, 1940). Los tejidos se observaron con un microscopio óptico marca Carl Zeiss, modelo Star. Las fotografías de los tejidos fueron tomadas con una cámara digital Evolution a través de un microscopio Olympus Bx-52. Las primeras observaciones confirmaron que el tamaño no refleja claramente ningún estadio de maduración; por ello, los botones florales se reagruparon con base en el desarrollo de los granos de polen. El primer estadio presentaba tapete abundante, el segundo con tétrades y tapete aún presente y el tercero con micrósporas y sin tapete. Aunque se observaron y compararon los botones florales en los 3 estadios de desarrollo, sólo se describieron los que estaban en etapa de micróspora, cuando el tapete se había degradado completamente.

\section{Resultados}

La anatomía de los botones florales de las especies estudiadas es similar, independientemente del estadio de desarrollo; únicamente varía la ornamentación en la cutícula de la epidermis adaxial del tubo del perianto que cambia de lisa a rugosa desde su porción basal hasta el ápice del mismo, en los botones que presentan micrósporas maduras y el tapete se ha consumido totalmente.

Tubo periántico. En todas las especies analizadas el tubo del perianto está fusionado en la base del ovario (Figs. 1A, 2A). En este nivel, en sección transversal, se presenta cutícula lisa y epidermis uniestratificada, con células rectangulares en sentido periclinal. Subyacente a la epidermis se encuentra un parénquima formado por células que disminuyen de tamaño hacia la región central del tubo del perianto, en ésta se observan células en actividad mitótica. En el parénquima se localizan haces vasculares colaterales, concéntricos; los externos han completado su desarrollo, los intermedios tienen crecimiento activo y los cercanos a la región central inician su diferenciación. Además, el parénquima presenta idioblastos con rafidios en todas las especies, pero son más abundantes en $P$. howardii (Fig. 1B). Tanto en secciones transversales como longitudinales, se observó que el tejido vascular se diferencia en sentido acrópeto y el haz es colateral (Fig. 1C).

En la base del estilo, el tubo del perianto se encuentra separado de la región distal del ovario. Tanto la epidermis, que también es uniestratificada, como el parénquima, son similares a la base del perianto, excepto que las células epidérmicas presentan pared periclinal externa más gruesa que la interna. En este nivel del tubo y por arriba del primer tercio, $P$. howardii presenta estomas.

En la región distal del tubo floral se observan los tépalos externos e internos. Los primeros tienen cutícula 
lisa en la superficie abaxial y estriada en la adaxial, mientras que los internos presentan cutícula estriada en ambas superficies (Fig. 1D). Además, la epidermis externa e interna en secciones transversal y longitudinal tiene células cuadradas, con pared periclinal externa más gruesa que la interna y papilas en el ápice de los tépalos (Fig. 1E). El parénquima situado entre ambas epidermis está constituido por células de tamaño y forma variados. Los haces vasculares terminan cerca del ápice de los tépalos.

Androceo. En la pared adaxial del tubo se encuentran protuberancias de parénquima con un haz vascular central, separadas del tubo en secciones localizadas hacia el ápice del mismo y constituyen la base de los 6 filamentos, dispuestos irregularmente alrededor del estilo (Fig.1. F, $\mathrm{G}, \mathrm{K}$ ). Los filamentos se originan en diferentes niveles del tubo floral. En $P$. geminiflora var. geminiflora y $P$. multicolor (Fig. 1H) ocurre por debajo de la región distal del ovario y en $P$. howardii en la región distal del mismo (Fig. 1I). En P. densiflora, P. nelsonii y P. platyphylla (Fig. $1 \mathrm{~J})$, los filamentos se originan en la región cercana a la base de los tépalos. En sección transversal, cada filamento es anchamente elíptico, con cutícula estriada (Fig. 1K, L) y epidermis uniestratificada. El tejido parenquimático presenta de 5 a 6 estratos de células; únicamente $P$. howardii tiene hasta 9 capas. Además, en todas las especies se observaron idioblastos con rafidios y un haz vascular colateral en la región central.

Las anteras tienen cutícula estriada y epidermis simple, con células en sección transversal comúnmente ovado-depresas, sólo en $P$. howardii son transversalmente elípticas en sentido periclinal y lobular-papilosas en P. multicolor (Fig. 1M). Por debajo de la epidermis se encuentra el endotecio formado por un estrato de células perpendiculares a la cavidad del saco polínico. Las tecas tienen 2 sacos polínicos cada una. En todas las especies estudiadas se observaron tétrades con arreglo tetragonal y polen maduro binucleado, uno de estos núcleos corresponde a la célula vegetativa y el otro a la generatriz. El tapete se observó previo a la formación de las micrósporas y es de tipo secretor. En $P$. howardii, $P$. geminiflora var. geminiflora, P. multicolor y $P$. nelsonii, se encontró polen fértil y abortivo principalmente. Este último se reconoció por la presencia de micrósporas obliteradas. El tejido conectivo está constituido por 2 capas de células, por debajo de éste, el parénquima tiene células con tamaño variable y haces vasculares colaterales.

Gineceo. En la parte media del ovario y en sección transversal se observan 3 carpelos y 3 lóculos. En la región abaxial, la cutícula es lisa y la epidermis con células rectangulares en sentido periclinal; la mayoría en actividad mitótica y pared periclinal externa más gruesa que la interna. El parénquima subyacente está constituido por células isodiamétricas, que disminuyen de tamaño hacia los lóculos, con rafidios y haces vasculares colaterales, éstos se distribuyen por todo el parénquima en forma circular y corresponden al segundo grupo de haces vasculares, presente desde la base del perianto. Estos haces se dividen 1 o 2 veces periclinalmente, por lo que su número varía en cada región de las paredes del ovario; de este modo, se localizan 2 haces frente a cada septo en la parte media del lóculo, 3 se alinean periclinalmente y, entre éstos, 2 más están dispuestos anticlinalmente (Fig. 2B).

Los septos que delimitan los lóculos se orientan radialmente y en el centro de cada uno se localizan los nectarios septales e interloculares. En $P$. howardii los nectarios son de igual o mayor longitud que los lóculos en sentido radial, diferentes de las otras especies estudiadas, donde los nectarios sólo abarcan $3 / 4$ partes de la longitud del lóculo. En ambos lados de los nectarios se presentan haces vasculares; éstos son continuación de los centrales, presentes desde la base del perianto (Fig. 2C).

La epidermis de los lóculos tiene generalmente células rectangulares con citoplasma denso (Fig. 2D), pero pueden ser cuadradas en la región basal y distal del lóculo. La placentación es axial. Los óvulos se organizan por pares, son bitégmicos, crasinucelados y anátropos (Fig. 2B, E, F). Los óvulos localizados en las zonas basal y distal del ovario son más pequeños y menos desarrollados en relación con los de la zona media, que muestran mayor tamaño y desarrollo en todas las especies.

Tanto en sección transversal como longitudinal la base del estilo tiene cutícula lisa y epidermis uniestratificada con células rectangulares en sentido periclinal. Polianthes howardii, $P$. multicolor y $P$. platyphylla, también presentan esta forma, pero orientadas anticlinalmente. Las características anatómicas del parénquima subyacente son las mismas descritas para la pared del ovario. En la región distal del ovario se observan 9 haces vasculares, 3 de mayor tamaño, próximos a la epidermis, localizados en los vértices de un triángulo y 6 menores en el centro. En $P$. multicolor y $P$. howardii solamente se observan los haces vasculares externos en este nivel (Fig. 2G). En sección transversal y longitudinal, la región distal del ovario presenta 3 canales estilares (Fig. 2G), su epidermis está formada por células rectangulares en sentido periclinal y las células que delimitan los nectarios septales son cuadradas (Fig. 2G, H). Debido a la fusión de los 3 canales estilares, en la parte media del estilo donde sólo se observa 1 canal estilar (Fig. 2J), la región abaxial presenta cutícula estriada. Los haces vasculares se disponen en 3 grupos al centro de los carpelos y las células epidérmicas del canal estilar presentan citoplasma denso con núcleos evidentes (Fig. 2I, J). El ápice del estilo en sección transversal, tiene forma triangular con vértices redondeados (Fig. 
2J) y es la zona donde terminan los haces vasculares (Fig. 2K, L). También en sección transversal, la región abaxial presenta cutícula estriada y células epidérmicas papilosas. Por debajo de la epidermis del canal estilar se encuentra el tejido de transmisión, con citoplasma denso y espacios intercelulares muy reducidos. En este extremo, la porción adaxial del estilo presenta cutícula lisa y epidermis anatómicamente igual a su parte media.

En sección transversal el estigma es trilobado, con lóbulos reniformes alargados, la región abaxial presenta cutícula estriada y la adaxial lisa. La epidermis adaxial tiene células papilosas con citoplasma denso, en $P$. howardii, además se presentan idioblastos con rafidios y el tejido de transmisión está distribuido por todo el estigma (Fig. 2M, N).

\section{Discusión}

Tubo periántico. Las características anatómicas del perianto en todos los botones florales observados son uniformes, excepto en la cutícula, que generalmente cambia de lisa a estriada con la madurez En los botones más jóvenes es lisa, probablemente porque el perianto se encuentra en crecimiento activo y no se ha acumulado cutina. Sin embargo, se observó que algunos en estadio intermedio y otros más desarrollados también presentan cutícula lisa en su región abaxial, desde la base del perianto hasta el inicio de los tépalos internos. A partir de esta zona y hasta donde se desarrollan las papilas del ápice de los tépalos externos (Fig. 1E), la cutícula es estriada, y de igual manera se presenta tanto en la cara externa como interna de los tépalos internos. Probablemente las papilas excretan alguna sustancia que atrae a los polinizadores. La presencia de papilas con cutículas estriadas, tal vez se relaciona con la pérdida de agua. Esta combinación de caracteres se encuentra en otros géneros relacionados con Polianthes, como Manfreda y Prochnyanthes.

Polianthes howardii es la única especie que en la base externa del tubo periántico presenta estomas; la presencia de éstos se relaciona con el color verde por arriba de su primer tercio. Seguramente en esta especie, los estomas no son funcionales y tampoco contribuyen en la movilización de fotosintatos. Una conclusión similar señalan Hew et al. (1980) en orquídeas, quienes comprobaron que los estomas florales son incapaces de realizar fotosíntesis, debido a que la conductancia del vapor de agua es menor al de las hojas y su transpiración en condiciones de luz y oscuridad es la misma.

Los haces vasculares del tubo periántico son colaterales y uniformes en cuanto a número y disposición. El único tipo de inclusiones minerales presentes son los rafidios; en este sentido, Prychid y Rudall (1999) registraron que estos cristales son muy comunes en las hojas de las agaváceas. Sin embargo, no se tenían registros de su presencia en las flores. Probablemente estas inclusiones contribuyan al sostén del tubo, pues las flores son muy crasas, o bien, como mecanismo de defensa a la herbivoría.

Androceo. Las observaciones realizadas indican que los filamentos se originan en diferentes niveles del tubo floral. Por ejemplo, en P. geminiflora var. geminiflora y $P$. multicolor (Fig. 1H) ocurre por debajo de la porción distal del ovario, y en la porción distal en $P$. howardi (Fig. 1I). Estas 3 especies pertenecen al subgénero Bravoa y apoyan su reconocimiento. Por otro lado, en $P$. densiflora, $P$. nelsonii y P. platyphylla (Fig. 1J), del subgénero Polianthes, los filamentos se originan en la región cercana a la base de los tépalos. Al reconocer la zona donde se dividen los haces que vascularizan cada uno de los filamentos, se pudo determinar el lugar de origen de los mismos como se ha descrito para otros géneros de monocotiledóneas (Rosa y Scatena, 2007).

En las descripciones morfológicas originales de todas las especies de los subgéneros Bravoa y Polianthes (Rose, 1903; Verhoek-Williams, 1976; Solano y García-Mendoza, 1998; García-Mendoza y Solano, 2007), se menciona que los filamentos, son largos o cortos, insertos en diferentes niveles del tubo, sin indicar el área en la que se originan. Por otro lado, Álvarez $(1987,1988)$ señaló que los géneros Polianthes, Pseudobravoa y Runyonia, poseen estambres con filamentos muy cortos que se insertan en la garganta del tubo floral, mientras que Bravoa y Prochnyanthes poseen estambres con filamentos largos insertos desde la parte basal del tubo floral; tampoco registró la zona de origen. Solano (2000) consideró que los filamentos están adnados al tubo del perianto desde su base y su longitud incluye la porción libre de los mismos. En este estudio se evidencia que los estambres se originan justo en el área del tubo periántico donde se insertan, y no están adnados al mismo desde su base.

El tipo de tapete registrado en las especies analizadas es secretor, el cual según Furness y Rudall (2001a) predomina en las angiospermas basales y se encuentra de manera uniforme en Asparagales, Dioscorales y Liliales. Este tipo de tapete también fue señalado por Joshi y Pantulu (1941) en $P$. tuberosa. Las tétrades de las especies estudiadas presentaron un arreglo tetragonal. Igual disposición fue indicada por Penet et al. (2005) en Asparagales superiores, donde se incluyen las agaváceas, aunque también se pueden encontrar disposiciones en forma linear o de "T". En este contexto, Joshi y Pantulu (1941) y Cave (1955) señalaron que $P$. tuberosa presenta estos últimos 2 arreglos de las tétrades, pero en este trabajo no se observaron. 


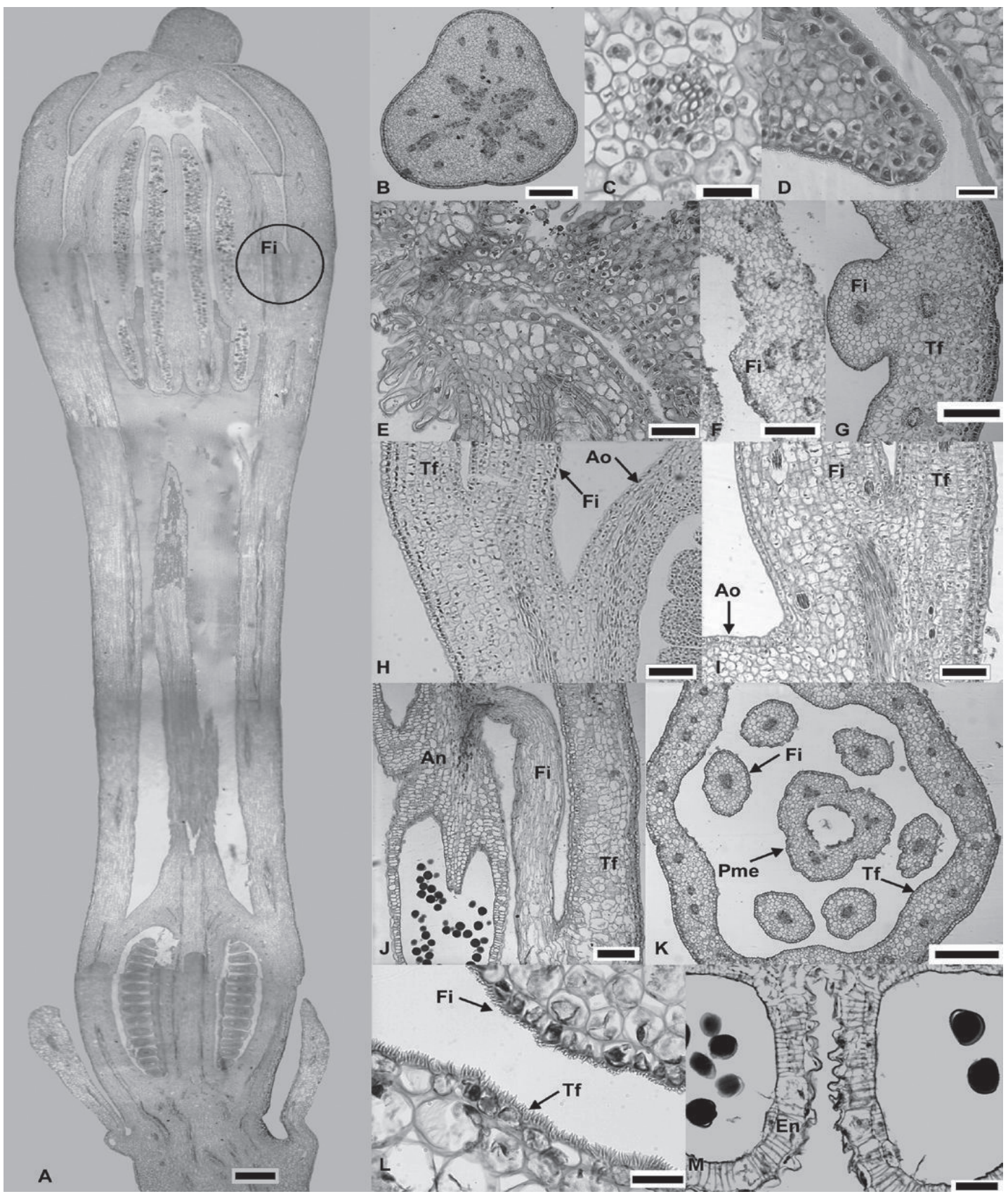

Figura 1. Secciones longitudinales y transversales de botones florales de Polianthes. A, Polianthes nelsonii, sección longitudinal de botón completo, filamento inserto cerca de los tépalos internos del tubo periántico. La inserción está señalada con un círculo. B-C. Secciones transversales. B, P. multicolor, base del perianto; C, P. nelsonii, haz vascular colateral del perianto. D-E. Secciones longitudinales; D, P. nelsonii, tépalo interno, epidermis y cutícula estriada; E, $P$. howardii, tubo floral, región apical. Cutícula estriada y epidermis papilosa. F-G. Secciones transversales. Ramificación de los haces vasculares de cada filamento. F, P. platyphylla; G, $P$. nelsonii. H-J. Secciones longitudinales. H, P. geminiflora var. geminiflora, filamento inserto por debajo de la región distal del ovario; I, $P$. howardii, filamento inserto a nivel de la región distal del ovario; J, P. densiflora, filamento inserto por debajo de los tépalos. K-M. Secciones transversales. K, P. multicolor, filamentos con forma anchamente elíptica; $\mathbf{L}, P$. nelsonii, filamento y tubo floral con cutícula estriada; M, P. multicolor, pared de la antera con epidermis uniestratificada papilosa, endotecio y granos de polen. An $=$ antera, Ao $=$ región distal del ovario, $\mathrm{En}=$ endotecio, $\mathrm{Fi}=$ filamento, $\mathrm{Pme}=$ parte media del estilo, $\mathrm{Tf}=$ tubo floral. $\mathrm{Barras}: \mathrm{A}=1 \mathrm{~mm} ; \mathrm{B}, \mathrm{G}, \mathrm{K}=300$ $\mu \mathrm{m} ; \mathrm{F}, \mathrm{J}=200 \mu \mathrm{m} ; \mathrm{I}=100 \mu \mathrm{m} ; \mathrm{C}, \mathrm{D}, \mathrm{M}=50 \mu \mathrm{m} ; \mathrm{H}, \mathrm{L}=30 \mu \mathrm{m} ; \mathrm{E}, \mathrm{K}=25 \mu \mathrm{m}$. 


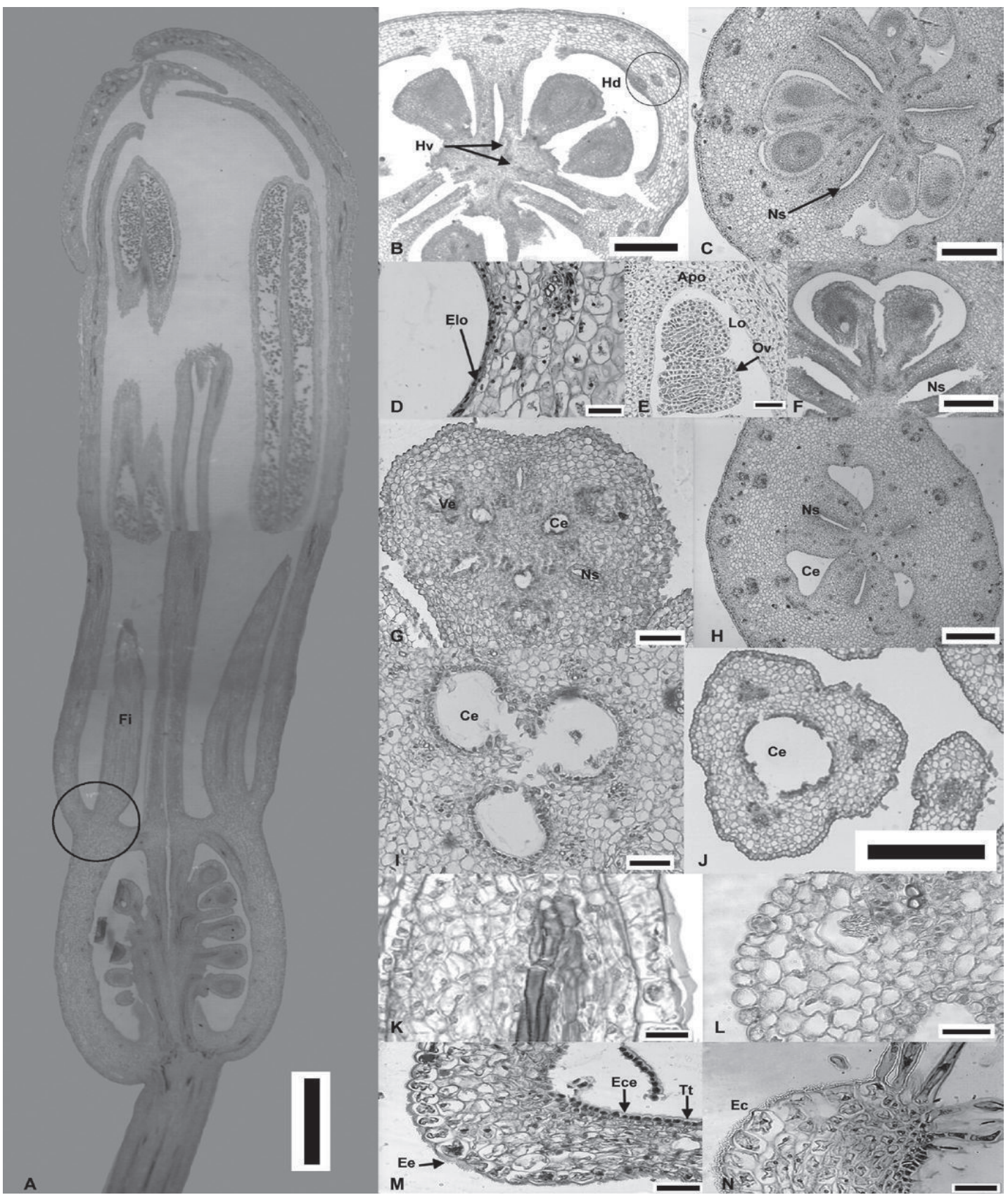

Figura 2. Secciones longitudinales y transversales de botones florales de Polianthes. A, Polianthes howardii, sección longitudinal, filamentos insertos en la región distal del ovario. La inserción está señalada con un círculo. B-D. Secciones transversales. B, P. multicolor, ovario trilocular; $\mathbf{C}, P$. howardii, región media del ovario, lóculos y nectarios septales-interloculares; $\mathbf{D}, P$. densiflora, epidermis del lóculo. E. P. geminiflora, sección longitudinal, lóculo con óvulos inmaduros; F-J. Secciones transversales. F, P. nelsonii, lóculo con óvulos bitégmicos, crasinucelados y anátropos; G, P. platyphylla, base del estilo con canales estilares y nectarios; H, $P$. howardii, nectarios septales; I, P. nelsonii, parte media del estilo, a este nivel una cavidad estilar; J, P. multicolor, parte distal del estilo, cavidad estilar; K, P. howardii, sección longitudinal, parte distal del estilo, región abaxial con cutícula lisa y terminación de un haz vascular. L-M. Secciones transversales. L, P. howardii, base del estigma, idioblasto con rafidios; M, P. nelsonii, estigma con cutícula estriada y tejido de trasmisión; N, P. geminiflora var. geminiflora, sección longitudinal, estigma con papilas. Apo = región distal del ovario, Ce = cavidad estilar, Ec = cutícula estriada, Ece = epidermis cavidad estilar, Ee = epidermis externa, Elo = epidermis locular, $\mathrm{Fi}=$ filamento, $\mathrm{Hd}=$ haces abaxiales, $\mathrm{Hv}=$ haces adaxiales, $\mathrm{Lo}=$ lóculo, $\mathrm{Ns}=$ nectario septal, interlocular, $\mathrm{Ov}=$ óvulo, $\mathrm{Tt}=$ tejido de trasmisión, Ve $=$ haz vascular adaxial. Barras: A = $1 \mathrm{~mm} ; \mathrm{B}, \mathrm{C}, \mathrm{F}, \mathrm{H}, \mathrm{J}=300 \mu \mathrm{m} ; \mathrm{G}=100 \mu \mathrm{m} ; \mathrm{D}, \mathrm{E}, \mathrm{I}, \mathrm{M}, \mathrm{N}=50 \mu \mathrm{m} ; \mathrm{L}=30 \mu \mathrm{m} ; \mathrm{K}=25 \mu \mathrm{m}$. 
De acuerdo con Furness y Rudall (2001b) y Penet et al. (2005) estas 3 disposiciones son indicativas de citocinesis sucesiva.

En los botones florales de menor tamaño localizados hacia la región distal de la inflorescencia de $P$. howardii, P. geminiflora var. geminiflora, P. multicolor y P. nelsonii, se observó polen abortivo. El mismo tipo de polen fue descrito por Nickolai et al. (2001) en Agave angustifolia y A. fourcroydes. Nickolai et al. (2001) registraron que Agave angustifolia es hexaploide y señalaron la presencia de polen abortivo. Probablemente estas especies de Polianthes sean poliploides, lo cual deberá confirmarse con estudios citológicos. Otra posible explicación sobre este tipo de polen es que su presencia pueda deberse a algunas aberraciones mitóticas y meióticas que ocurren durante la microsporogénesis, o al mal funcionamiento del tapete, ya que éste desempeña un papel importante durante el proceso y en la maduración de los granos de polen (Chapman, 1987). Por otro lado, como la inflorescencia de todas las especies del género estudiado presenta maduración acrópeta, probablemente las sustancias de reserva no son suficientes para desarrollar micrósporas funcionales en las flores apicales.

Gineceo. Anatómicamente el gineceo de las 6 especies analizadas también es uniforme en epidermis, estilo, estigma, placentación y óvulos. Los nectarios, de acuerdo con la clasificación de Dahlgren y Clifford (1982), basados en el trabajo de Daumann (1970), son septales, característicos de la familia Agavaceae. Schmid (1985, 1988) indicó que este tipo de nectarios se conoce como liliado (lilid), el más común dentro de las monocotiledóneas. De acuerdo con la clasificación de Simpson (1993), por su posición en relación con los lóculos, los nectarios de las 6 especies estudiadas son interloculares y se extienden en 5 de ellas hasta la zona próxima al estigma, donde se localiza su abertura como se ha registrado en la mayoría de las Asparagales (Álvarez, 1988; Smichd, 1988). Polianthes howardii es una excepción, pues la abertura está en la región distal del ovario, la misma situación fue registrada por Pantil y Pai (1985) en P. tuberosa. En las especies estudiadas la longitud de los nectarios septales en sentido radial abarca $3 / 4$ partes de la longitud del lóculo y sólo en $P$. howardii iguala su tamaño..

Todas las especies analizadas presentan óvulos anátropos, bitégmicos y crasinucelados, así como placentación axial, como ya se ha registrado para otras especies del género Polianthes y otras Agavaceae (Joshi y Pantulu, 1941; Rudall, 1997; Verhoek-Williams, 1998). De acuerdo con Palser (1975), los caracteres bitégmico y crasinucelado son plesiomórficos en las angiospermas.

En conclusión, la anatomía de las especies del género Polianthes es muy homogénea; por ello se considera que el único carácter anatómico que sustenta la división del género en 2 subgéneros, Bravoa y Polianthes, es la vascularización de los filamentos estaminales. En el subgénero Bravoa los cordones vasculares de los filamentos divergen en la base del tubo periantal, la inflorescencia es un racimo, las flores generalmente son de color diferente al blanco y sin fragancia. En el subgénero Polianthes los cordones vasculares de los filamentos se dividen por debajo de los tépalos, las inflorescencias son espigas con flores blancas $\mathrm{y}$ fragantes.

\section{Agradecimientos}

Al Consejo Nacional de Ciencia y Tecnología por la beca (Núm. 198023) otorgada al primer autor para sus estudios de maestría en ciencias. C. Correa D. nos brindó su compañía durante las recolectas por toda el área de distribución del género, Hilda Flores Olvera hizo importantes aportaciones durante este estudio. Sonia Vázquez S. y un revisor anónimo también realizaron comentarios al manuscrito. Julio César Montero R. digitalizó las figuras.

\section{Literatura citada}

Álvarez, A. 1987. Sistemática y filogenia de la familia Agavaceae Endlicher. Tesis doctorado, Facultad de Biología, Jardín Botánico Nacional. Universidad de La Habana. 210 p.

Álvarez, A. 1988. Morfología y anatomía floral de las Agavaceae. Revista del Jardín Botánico Nacional 9:37-57.

Cave, M. S. 1955. Sporogenesis and the female gametophyte of Phormium tenax. Phytomorphology 5:247-253.

Chapman, G. P. 1987. The tapetum. Pollen: cytology and development. International Review of Cytology 107:111125.

Dahlgren, R. y H. T. Clifford. 1982. The monocotyledons: a comparative study. Academic, London. 210 p.

Dahlgren, R. M. T., H.T. Clifford y P.F. Yeo. 1985. The families of the monocotyledons. Structure, evolution, and taxonomy. Springer-Verlag, New York. 520 p.

Daumann, E. 1970. Das Blutennektarium der Monocotyledonen unter besonderer Berücksichtigung seiner sytematischen und phylogenetischen Bedeutung. Feddes Repertorium 80:463590.

Espejo, S. A. y A. R. López F. 1992. Las monocotiledóneas mexicanas. Una sinopsis florística. Parte I. Consejo Nacional de la Flora de México. Universidad Autónoma MetropolitanaIztapalapa. México, D. F. 76 p.

Furness, C. A. y P. J. Rudall. 2001a. The tapetum in basal 
angiosperms: early diversity. International Journal of Plant Sciences 162:375-392.

Furness, C. A. y P. J. Rudall. 2001b. Pollen and anther characters in monocot systematics. Grana 40:17-25.

García-Mendoza, A. y E. Solano. 2007. Polianthes oaxacana y P. geminiflora var. pueblensis (Agavaceae), taxa nuevos de México. Acta Botanica Mexicana 78:111-123.

García-Mendoza, A. y R. Galván V. 1995. Riqueza de las familias Agavaceae y Nolinaceae en México. Boletín de la Sociedad Botánica de México. 56:7-24.

Hew, C. S., G. L. Lee y S. C. Wong. 1980. Occurrence of nonfunctional stomata in the flowers of tropical Orchids. Annals of Botany 46:195-201.

Johansen, D. A. 1940. Plant microtechnique. McGraw Hill, New York. 523 p.

Joshi, A. C. y J. V. Pantulu. 1941. A morphological and cytological studies of Polianthes tuberosa Linn. Journal of the Indian Botanical Society 20:37-71.

Nickolai, M. P., F. A. Barredo-Pool., I. C. Borges-Argáez., M. A. Herrera-Alamillo, A. Mayo-Mosqueda, J. L. Herrera-Herrera y M. L. Robert. 2001. Reproductive biology of henequen (Agave fourcroides) and its wild ancestor Agave angustifolia (Agavaceae). I. Gametophyte development. American Journal of Botany 88:1966-1976.

Palser, B. F. 1975. The bases of angiosperm phylogeny: embryology. Annals of the Missouri Botanical Garden 62:621-646.

Pantil, D. A. y R. M. Pai. 1985. The nectaries in the Agavaceae. Acta Botanica Indica 13:289-291.

Penet, L., S. Nadot, A. Ressayre, A. Forchioni, L. Dreyer y P. H. Gouyon. 2005. Multiple developmental pathways leading to a single morph: Monosulcate pollen (Examples from Asparagales). Annals of Botany 95:331-343.

Prychid, Ch. J. y P. J. Rudall. 1999. Calcium oxalate crystals in monocotyledons: A review of their structure and systematics. Annals of Botany 84:725-739.

Rosa, M. M. y V. L. Scatena. 2007. Floral anatomy of Paepalanthoideae (Eriocaulaceae, Poales) and their nectariferous structures. Annals of Botany 99:131-137

Rose, J. N. 1903. Studies of Mexican and Central American Plants. Contributions from the United States National Herbarium 3:1-55.

Rudall, P. J. 1997. The nucellus and chalaza in monocotyledons: structure and systematics. Botanical Review 63:140-181.

Schmid, R. 1985. Funcional interpretations of the morphology and anatomy of septal nectaries. Acta Botanica Neerlandica 34:125-128.

Schmid, R. 1988. Reproductive versus extra-reproductive nectaries-historical perspective and terminological recommendations. Botanical Review 54:179-232.

Simpson, M. G. 1993. Septal nectary anatomy and pylogeny of the Haemodoraceae. Systematic Botany 18:593-613.

Solano, C. E. 2000. Sistemática del Género Polianthes L. (Agavaceae). Tesis, doctorado Facultad de Ciencias, Universidad Nacional Autónoma de México, México, D. F. 291 p.

Solano E. y T. P. Feria. 2007. Ecological niche modeling and geographic distribution of the genus Polianthes L. (Agavaceae) in Mexico: using niche modeling to improve assessments of risk status. Biodiversity and Conservation 16:1885-1900.

Solano, C. E. y A. García-Mendoza. 1998. Una nueva especie de Polianthes del estado de Oaxaca. Sida 18:97-101.

Verhoek-Williams, S. 1976. Polianthes howardii (Agavaceae): a new species from Colima, México. Phytologia 34:365-368.

Verhoek-Williams, S. 1998. Agavaceae. In The families and genera of vascular plants, vol. III. Flowering plants monocotyledons Lilianae (except Orchidaceae), K. Kubitzki (ed.). Springer-Verlag, Berlin. p. 60-70. 